\title{
Forward and Inverse Kinematics Applied to the Parallel Robot's Structure
}

\author{
Olaru D. Adrian, Olaru A. Serban, and Mihai C. Niculae
}

\begin{abstract}
The optimization in Robotics are applied to increase the 3D space precision, reduce the vibrations, choose the optimal applied base point of the robots to minimize the time of work, reduce electricity consumption, reduce the vibration and finally optimize the dynamic behavior. In the work was developed some proper algorithms to obtain, iteratively the extreme performances to the 3D space trajectory without vibration in the desirable field of the Fourier spectrum for serial and parallel robots. The research show haw can be increased the accuracy of the internal coordinate by solving the inverse kinematics with proper method with the goal to touch the extreme precision with less than $0.001 \mathrm{~mm}$. In the assisted researching of the dynamic behavior in mechatronics the important roles play modeling, simulation and optimization with virtual LabVIEW ${ }^{\mathrm{TM}}$ instrumentation. The paper proposes one assisted method solving the forward and inverse kinematics with the goal to minimize the final end-effecters trajectory errors, by optimizing the distance between the final position of the end-effecter and the proposed target. The proper virtual LabVIEW instrumentation used in the assisted research open the way to some other applications like: programming the collaborative robots, exoscheletons, parallel robots and multirobots applications. The proposed method was applied to many types of the robot's structures. Virtual instrumentation easily provides comparison between theoretical and experimental results and establishes the conditions to adjust and validate the proposed mathematical models. The paper shows numerous virtual instruments and some case study to optimize the motion of parallel robots end-effecters by the assisted simulation and animation after solving the inverse kinematics.
\end{abstract}

Index Terms-Assisted method, animation, forward kinematics, inverse kinematics, LabVIEW instrumentation, modeling, simulation.

\section{INTRODUCTION}

For the purpose of modeling, searching, animation and validating the mathematical model of the complex robotic structure, in the laboratory of the Dynamic Behavior of Industrial Robots of the faculty I.M.S.T.-U.P.B., there was designed and realized one arm type robot with five degrees of freedom. In a research stand, as it is presented in figure 1, can distinguish the following elements: robot with DC motors and encoders; stabilized source of continuous voltage; accelerometer; GekoDrive with PWM and $\mathrm{H}$ bridge;

Manuscript received September 15, 2017; revised December 12, 2017. This work was supported in part by the Machines Tools and Manufacturing Systems Department from IMST faculty, University Politehnica of Bucharest, Romania.

A. D. Olaru is with the University Politehnica of Bucharest, Machines and Manufacturing Systems Department, RO 60400 Romania (e-mail: aolaru_51@ymail.com).

S. A. Olaru was with RomSys Mechatronic Private Company, RO 013713, Romania (e-mail: serban1978@yahoo.com).

N. C. Mihai is with TechnoAccord Private Company on CNC Machines Tools and Robotics, CA 4170, Canada (e-mail: mniculae@yahoo.com). amplifying block; screen; computer; multimeter; connectors and the acquisition board. This stand with acquisition board is used to determinate exactly the characteristics of relative angular velocity and acceleration, the acceleration time and verify the precision of the relative displacement in the joints. Was tested some proper method for command files to control, by using the forward and inverse kinematics together with the Bipolar Sigmoid Hyperbolic Tangent Neural Network with Time Delay and Recurrent Links (BSHTNN-TDRL), the movement of the end-effecter, to touch the proposed space target with the precision better than $0.001 \mathrm{~mm}$. For the parallel structure was used one platform in the triangle form with three legs of the similar robot like this.

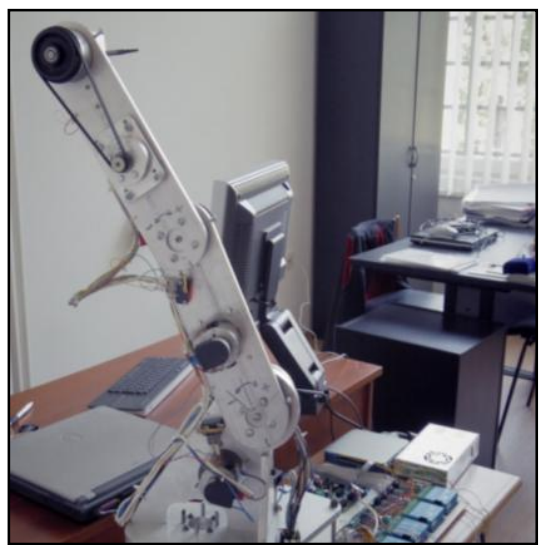

Fig. 1. The didactical arm type robot used in the assisted research of the robot's kinematics.

The paper shown one other way for assisted analyze of the kinematics positions in the parallel robot's structure by using the complex matrix method for the forward and inverse kinematics and the iterative proper method Iterative Pseudo Inverse Jacobian Matrix- Method coupled with Bipolar Sigmoid Hyperbolic Tangent Neural Network with Time Delay and Recurrent Links (IPIJMM- BSHTNN- TDRL).

Inverse Kinematics (IK) is a more important problem to solve in robotics more over if the number of degree of freedom (DOF) of the studied robot is bigger than 3. In the literature have been proposed many methods to solve it including: Gradient Method (GM), Jacobian Matrix Method (JMM), Pseudo-Inverse Jacobian Matrix Method (PIJMM), Gradient Projection Method (GPM), Inverse Jacobian Inertia Matrix Method (IJIMM), Neural Network Coupled Methods with some other numerical methods (NNCM) and other compound methods [1]-[12]. To develop the complex coupled method were researched the design of the neural network and the influences of his parameters to the convergence process [13]-[17]. In [18] the Iterative Pseudo Inverse Jacobian Matrix Method was combined with a Sigmoid Bipolar Hyperbolic Tangent Neural Network with 
Time Delay and Recurrent Links Method (IPIJMMSBHTNN- TDRL) for controlling only one robot movement with extreme precision better than $0.001 \mathrm{~mm}$.

\section{FORWARD AND INVERSE KINEMATICS}

\section{A. Criteria Index for Manipulability of Robots}

In the related literatures [19-27] were introduced some different indices. One of them was the dexterity of the robot. Other one is called kinematic manipulability, $\Delta$ that is proposed in [24] as the square root of the determinant of $\mathrm{JJ}^{\mathrm{T}}$. Since Jacobian $(\mathrm{J})$ is configuration dependent, kinematic manipulability is a measure of the local performance, which also gives an indication of how close the end- effecter to the singularity. For instance, means a singularity configuration, and therefore we wish to maximize the manipulability index to avoid singularities. Another usually used index is the condition number $k$ of Jacobian matrix, recommended in [25].

Global Dexterity Index (GDI) are given in [24] by:

$$
\mathrm{GDI}=\frac{\int_{V}(1 / \kappa) \mathrm{d} V}{V},
$$

where: $V$ is the total volume of workspace and $k$ denotes the condition number of Jacobian.

Jacobian condition number can be defined as [25]:

$$
\kappa=\|\mathbf{J}\|\left\|\mathbf{J}^{-1}\right\|,
$$
[26]:

The manipulability $\Delta$ of one robot was defined as follows

$$
\Delta=\sqrt{\operatorname{det}\left(\mathbf{J J}^{\mathrm{T}}\right)} .
$$

For example the manipulability $\Delta$ it is normalized by the workspace size and therefore gives a measure of kinematic performance, independent of the differing workspace volume of design candidates. The condition number $k$ is bounded between 0 and 1 and tends to infinity at singularities; hence, during numerical integration, the number of sample points near singularities has a reduced impact on the result since $k$ approaches zero at those points [27].

\section{B. Forward Kinematics in Robotics}

The forward kinematics we have used the matrix method presented in (4) and a versatile VI that can be very easily set.

$$
\left(r_{i}^{0}\right)=\left(r_{i-1}^{0}\right)+\left[D_{i-1}^{0}\right]\left(r_{i}^{i-1}\right)
$$

where $\left(r_{i}^{0}\right)$ is the column matrix of the current position vector, $\left(r_{i-1}^{0}\right)$ is the column matrix of the position vector of the previous point, $\left[D_{i-1}^{0}\right]$ is the $3 \times 3$ transformation matrix from $i-1$ to the base plane 0 , and $\left(r_{i}^{i-1}\right)$ is the column matrix of relative position between the points $i$ and $i-1$.

$$
\left(\bar{w}_{i, 0}^{i}\right)=\left[T_{i, i-1}\right] \cdot\left(\bar{w}_{i-1,0}^{i-1}\right)+\left(\bar{w}_{i, i-1}^{i}\right)
$$

where: $\left(w_{i, 0}{ }^{(i)}\right)$ is dual column matrix vector for absolute velocity $i$ joint reduced to plane $(i) ;\left(w_{i-1,0}{ }^{(i-1)}\right)$ - dual column matrix vector for absolute velocity $i-1$ joint reduced to plane $(i-1) ;\left[T_{i, i-1}\right]$ - quadratic matrix $6 \times 6$ for transfer velocity vector from plane $i-1$ to $i ;\left(w_{i, i-1}{ }^{(i)}\right)$ is dual column matrix for relative velocity vector between $i$ joint and $i-1$. Dual velocity vector have the angular and linear velocity, scalar have six rows.

Iterative vector matrix equation for dual acceleration are given by:

$$
\left(\bar{A}_{i, 0}^{(i)}\right)=\left[T_{i, i-1}\right] \cdot\left(\bar{A}_{i-1,0}^{(i-1)}\right)+\left(\bar{A}_{i, i-1}^{(i)}\right)
$$

where: $\left(A_{i, 0}{ }^{(i)}\right)$ is dual column matrix vector for absolute acceleration joint $i$ reduced to plane $(i) ;\left(A_{i-1,0}{ }^{(i-1)}\right)$ - is dual column matrix vector for relative acceleration between joint $i$ and $i-1$, reduced to plane $(i)$. Dual acceleration vector have as angular and linear acceleration vector of joints. Scalar column matrix expression for relative dual acceleration vector are given by:

$$
\left(A_{i, i-1}^{(i)}\right)=\left|\frac{\left(\varepsilon_{i, i-1}^{i}\right)+\left[\hat{\omega}_{i-1,0}^{i}\right] \cdot\left(\omega_{i, i-1}^{i}\right)}{\left(a_{i, i-1}^{i}\right)+\left[\hat{\omega}_{i-1,0}^{i}\right]^{2} \cdot\left(r_{i}^{0}\right)+2\left[\hat{\omega}_{i-1,0}^{i}\right] \cdot\left(v_{i, i-1}^{i}\right)}\right|
$$

absolute acceleration joint $i$ - 1 reduced to plane $i-1 ;\left(A_{i, i-1}{ }^{(i)}\right)$ is dual relative acceleration matrix vector.

For multiple robots application with different base point of robot the equation is given by:

$$
\begin{gathered}
R_{\psi, \theta, \varphi}=\left[\begin{array}{ccc}
C_{2} C_{3} & -C_{2} S_{3} & S_{2} \\
C_{1} S_{3}+S_{1} S_{2} C_{3} & C_{1} C_{3}-S_{1} S_{2} S_{3} & -S_{1} C_{2} \\
S_{1} S_{3}-C_{1} S_{2} C_{3} & S_{1} C_{3}+C_{1} S_{2} S_{3} & C_{1} C_{2}
\end{array}\right] \\
\left(\begin{array}{c}
1 \\
r_{r i l 1}^{0}
\end{array}\right)_{G W C}=\left[\begin{array}{cc}
1 & (0) \\
\left(T_{X, Y, Z}\right) & {\left[R_{\psi, \theta, \theta}\right]}
\end{array}\right]_{L W C 1}^{G W C}\left[\begin{array}{cc}
1 & (0) \\
\left(T_{X, Y, Z}\right) & {\left[R_{\psi, \theta, \theta}\right.}
\end{array}\right]_{R I I}^{L W C 1}\left(\begin{array}{c}
1 \\
r_{i}^{0}
\end{array}\right)_{R I I 1}
\end{gathered}
$$

where is introduced the global and local world coordinates are denoted by GWC and LWC, respectively; $\left[R_{\psi, \theta, \varphi}\right]$ is the Euler rotation angles matrix versus the robot's Cartesian system or of the LWC Cartesian system; $\left(T_{\mathrm{xyz}}\right)$ is the column translation matrix versus the robot's base Cartesian system or to the LWC Cartesian system.

\section{Inverse Kinematics in Robotics}

The Inverse Kinematics (IK) algorithm content the relations of the IPIJMM (10) and relations (11) of the neural network BSHTNN-TDRL.

$$
\begin{aligned}
& \left(d q_{i}\right)=\alpha\left[\left(T_{i}\right)-\left(F K\left(q_{i}\right)\right)\right]\left[J\left(q_{i}\right)\right]^{T}\left\{\left[J\left(q_{i}\right)\right]\left[J\left(q_{i}\right)\right]^{T}\right\}^{-1} \\
& \left(q_{j}\right)=\left(q_{i}\right)+\left(d q_{i}\right) \\
& (n)_{1}=[\underbrace{[w]^{1}}_{p_{1}}+\underbrace{\left(t c g_{1}\right)}_{p_{2}} \cdot\left(\varepsilon_{1}\right)]\left((p)-\left(a_{2}\right)\left((t)-\left(p_{3}\right)+1\right)\right)+\left(\left(b_{1}\right)+\left(\varepsilon_{1}\right)\right) ; \\
& \left(a_{1}\right)=\frac{\left(p_{4}\right)\left(1-e^{-n_{1}}\right)}{1+e^{-n_{1}}} ;\left(\varepsilon_{1}\right)=\left(t_{1}\right)-\left(a_{1}\right) \\
& n_{2}=[w^{2}+\underbrace{t c g_{2}}_{p 5} \cdot \varepsilon_{2}]\left(a_{1}\left(t-p_{6}+1\right)\right)+\left(b_{2}+\varepsilon_{2}\right) ; \\
& a_{2}=\frac{p_{7}\left(1-e^{-n_{2}}\right)}{1+e^{-n_{2}}} ;\left(\varepsilon_{2}\right)=\left(t_{2}\right)-\left(a_{2}\right) \\
& \left(q_{i}\right)=\left(p_{8}\right)\left(\left(a_{2}\right)-\left(\varepsilon_{f}\right)\right) ;\left(\varepsilon_{p o s}\right)=\left(t_{3}\right)-\left(r_{i}\right) ; \\
& \left.\left(n_{3}\right)=[\left[w^{3}\right]+\underbrace{\left(t c g_{2}\right.}_{p_{5}}) \cdot\left(\varepsilon_{p o s}\right)\right]\left(q_{i}\right)+\left(\left(b_{3}\right)+\left(\varepsilon_{p o s}\right)\right) ; \\
& \left(a_{3}\right)=\frac{\left(p_{9}\right)\left(1-e^{-n_{3}}\right)}{1+e^{-n_{3}}} ;\left(\varepsilon_{f}\right)=\left(t_{2}\right)-\left(a_{3}\right) .
\end{aligned}
$$

where: $\left(q_{\mathrm{i}}\right)$ - internal relative coordinates matrix; $d q_{\mathrm{i}}-$ 
increment of the internal robot's joints coordinates; $(F K)$ column matrix of the forward kinematics results; $\left[J\left(q_{i}\right)\right]^{T}\left\{\left[J\left(q_{i}\right)\right]\left[J\left(q_{i}\right)\right]^{T}\right\}^{-1}$ - pseudo inverse Jacobian matrix; $\alpha$

- convergence step of the iteration (teaching gain); $\left(t_{i}\right)$ column matrix of the target data of each layer; $\left(a_{i}\right)$ - matrix of output data of the sensitive sigmoid function; $\left(n_{i}\right)$ - matrix of input of the sensitive sigmoid function; $\left(\varepsilon_{i}\right)$ - matrix error after each layer; $t$-step delay; $\left(p_{i}\right)$ - parameter of the neural network what can be changed for optimizing the results; [ $w^{i}$ ]- weight matrix what can be on-line changed; $\left(b_{i}\right)$ -

biases matrix what can be on-line changed.

\section{Modeling, Simulation AND ANimation}

This stage of the research contents the FK model what was transformed to be animate the movement. For that each position of the joints was incremented by the length to be possible change the position by moving the angular relative value from each joint.

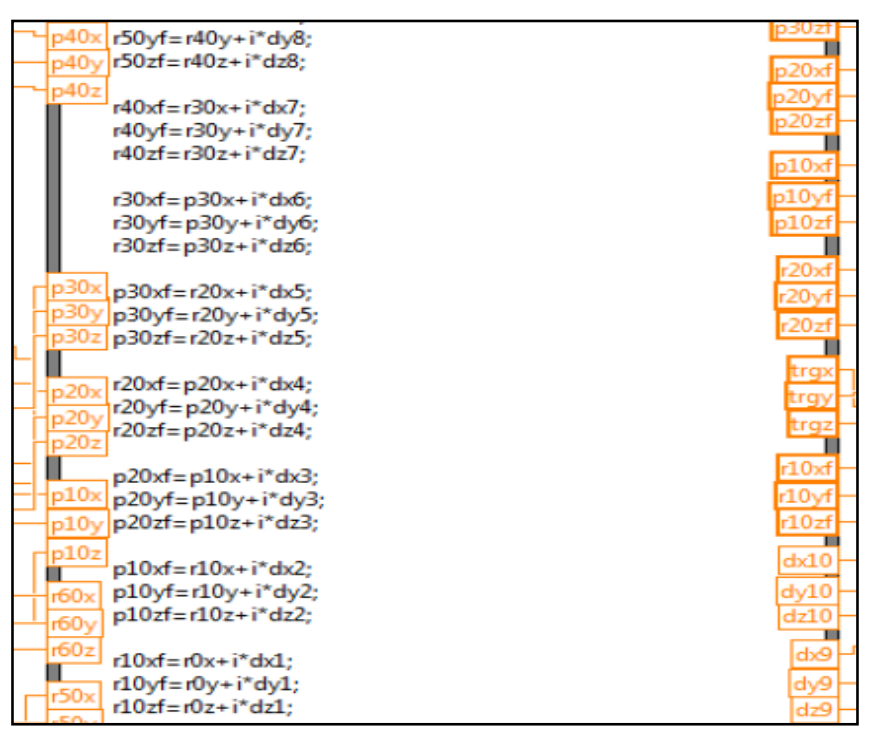

Fig. 2. Part of the LabVIEW block schema of the calculus of all incremental positions of the robots joints.

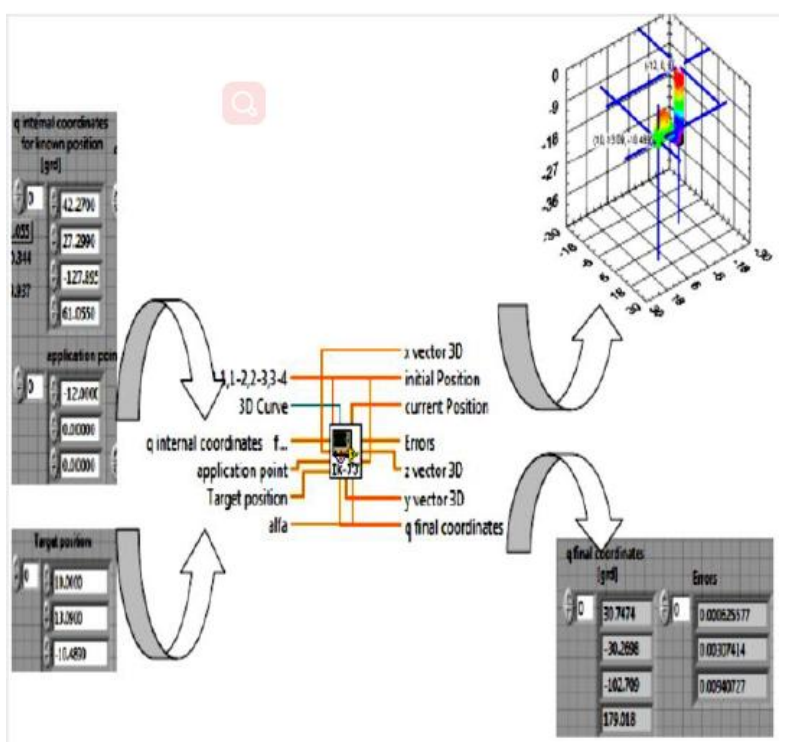

Fig. 3. The block schema of the algorithm to calculate the iterative position of the end-effecter and establish the better solution of the IK.

The algorithm work iteratively. Firstly compare the current position that was defined with the target position. After that adjust the current position of all joints, by using the IK relations (10) and (11). After one new comparison with the target will be established one new increment $d q_{i}$ what will adjust again the end-effecter position. The incremental process will continue before the target will be touch with imposed extreme precision, better than $0.001 \mathrm{~mm}$. The neural network ensures a fine adjustment of the increment near the target.

\section{STUdy CASE- PARALlEl Robot's STRUCTURE}

The proposed algorithm (Fig. 4) contains the following modules: the module of the transformation of the platform points about imposed rotations and translations about ox, oy and oz axes; the module to solve the IK with complex algorithm BSHTNN-TDRL with IPIJMM with constraints of the errors for all three robots better than $0.001 \mathrm{~mm}$; if the errors become larger than some imposed amounts, the obtained internal coordinates are exported to the neural network module to reduce them; this process works iteratively and stops when the errors are less than their imposed amounts and the values of the internal coordinates will be written on the external file together with the required transformations values; animation module that animate the movements in the space of the closed robot's structure by reading the file with transformation in the space of the platform and internal coordinates; the process can be resumed if a new position is required. By using this program can be written the file for the movement in the space of the platform in one closed structure.

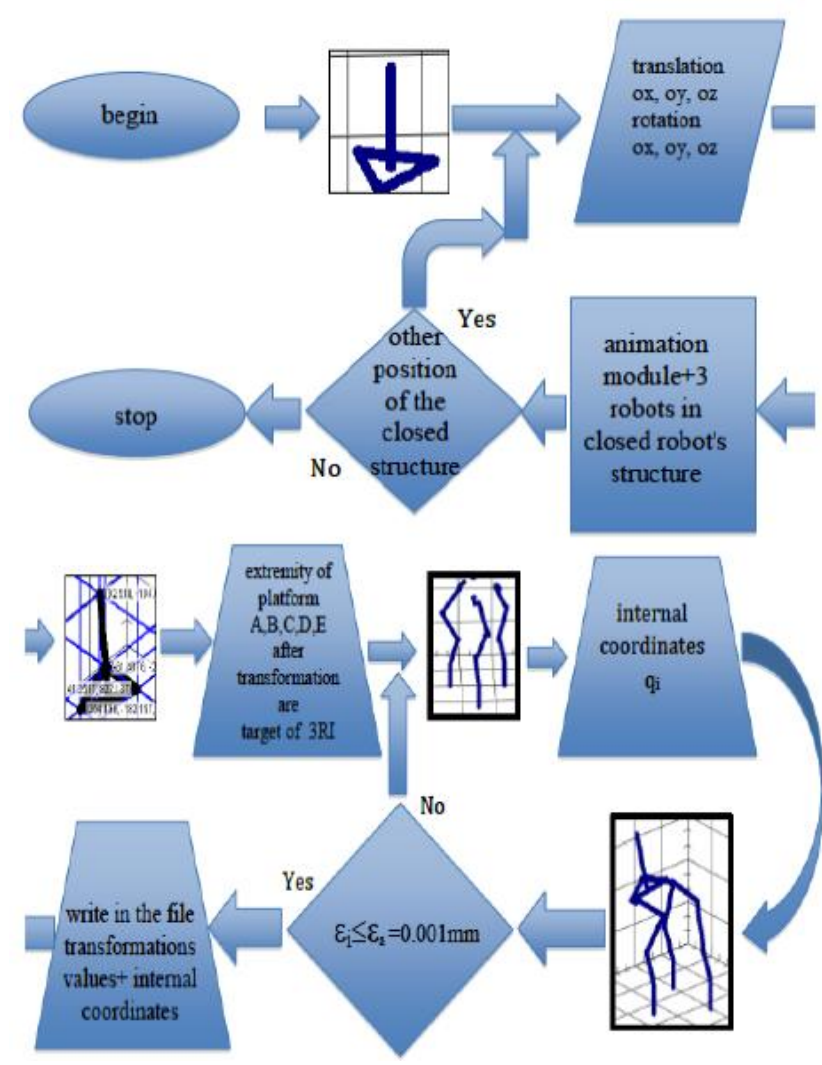

Fig. 4. Block schema of the complex program for the parallel robot's structure with triangle platform.

Applying this algorithm will be possible to designed all robot's parallel structure and check off-line the space application of end-effecter to determine the manipulability, 
the singular points or GDI.
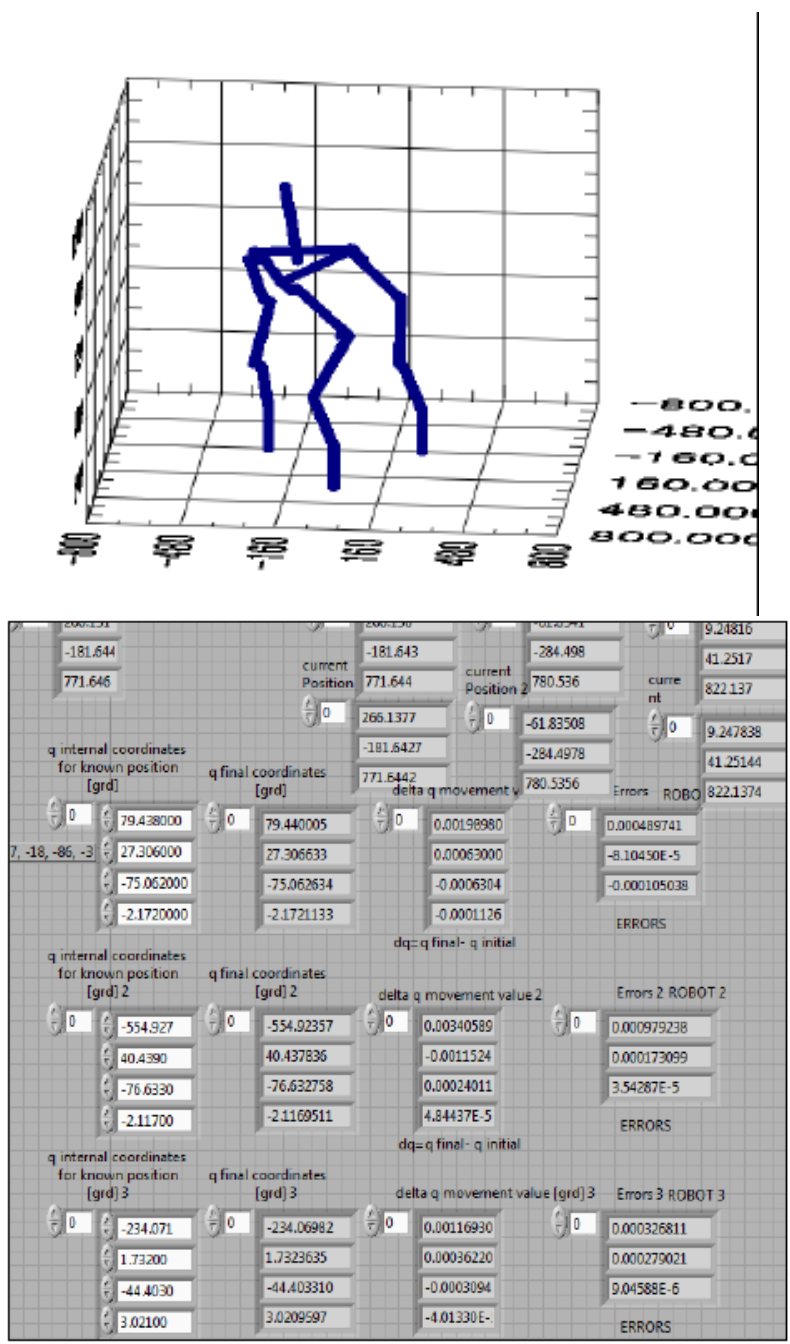

Fig. 5. The front panel of the complex algorithm for the parallel robot's structure.

\section{CONCLUSION}

After analyzing the obtained results of the assisted simulation and experiments, we can make the following remarks: (i) was established and validated one complex matrix method to solve the Forward kinematics (FK) and also the Inverse Kinematics (IK); (ii) has been achieved the assisted experimental research by using data acquisition board and didactical arm type robot and has been checked the proposed matrix model for the robot's kinematics; (iii) has been achieved some virtual $\mathrm{LabVIEW}^{\mathrm{TM}}$ instrumentations for the data acquisition, simulation and animation and for the iterative assisted research of the inverse kinematics. All these steps open the way to the smart control of the parallel robot's structure; (iv) also has been designed one complex VI-s that contains many other LabVIEW ${ }^{\mathrm{TM}}$ instruments that assure very easily to program one parallel robot structure with extreme precision better than $0.001 \mathrm{~mm}$; (v) the method of the simulation and experimental scenario of the robots' TCP movements along a known target curve $\boldsymbol{\Gamma}$ via $\mathrm{LabVIEW}^{\mathrm{TM}}$ in the space, enabled us to analyze and optimize the off-line control of the motors' movement; (vi) the designed method and the proposed and validated program can be used off-line in many other parallel robot application; (vii) by applying the new defined indexes criteria like $G D I, k$, and $\Delta$ will be possible to control better the manipulability and singularity of all three used robots in the complex closed structure; (viii) the animation program confirm the results of the applied algorithm and also the assisted applied method; (ix) this work open the way to the construction and optimizing the space movements of the parallel robot's structure and the design of the closed robot's structure with platform with three legs robots; (x) all created LabVIEW VI-s are generally and they can be used in many other application when it is necessary to obtain the extreme precision and accuracy, see figs. 7-9; (xi) the proposed method, the algorithm and the LabVIEW ${ }^{\mathrm{TM}} V I$-s can be applied in many other application of the complex open and closed robot's structures and also in collaborative applications when will be appeared workers and the security systems for workers.

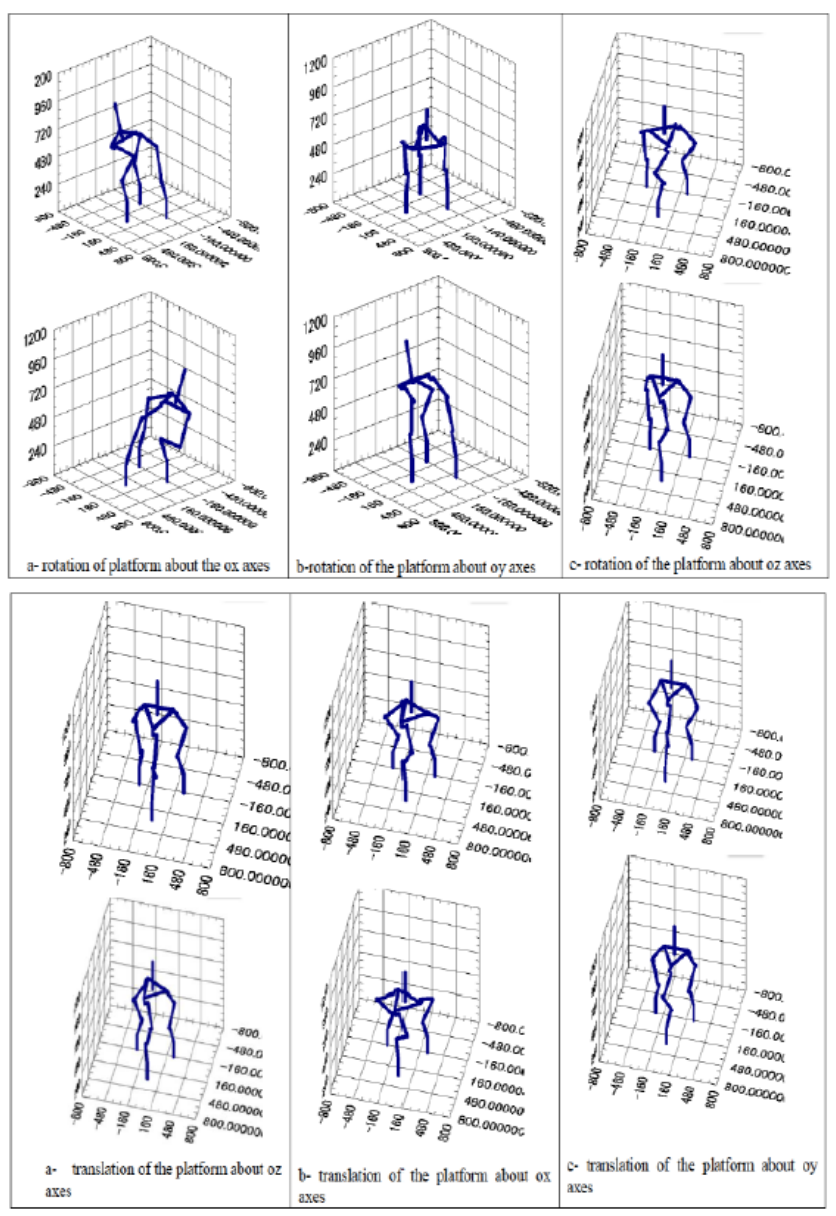

Fig. 6. Some examples with the parallel robot's structure with the rotations and translations about ox, oy and oz axes.

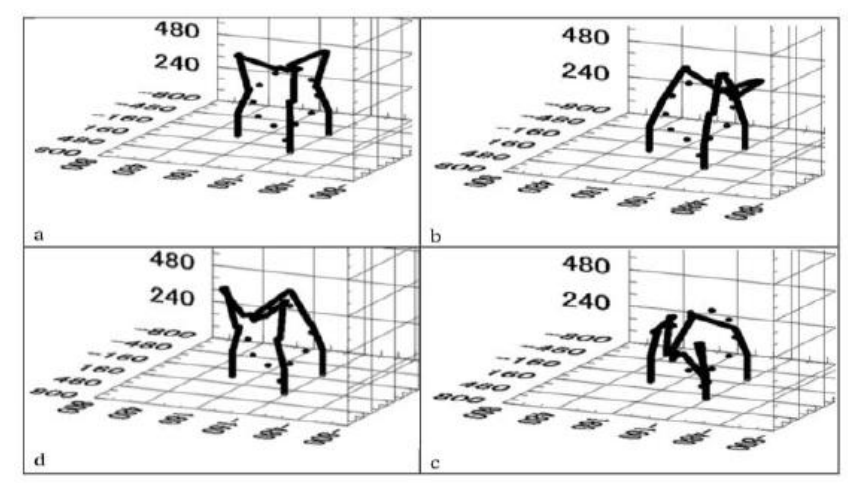

Fig. 7. Animation of the parallel robot structure with only one common point. 


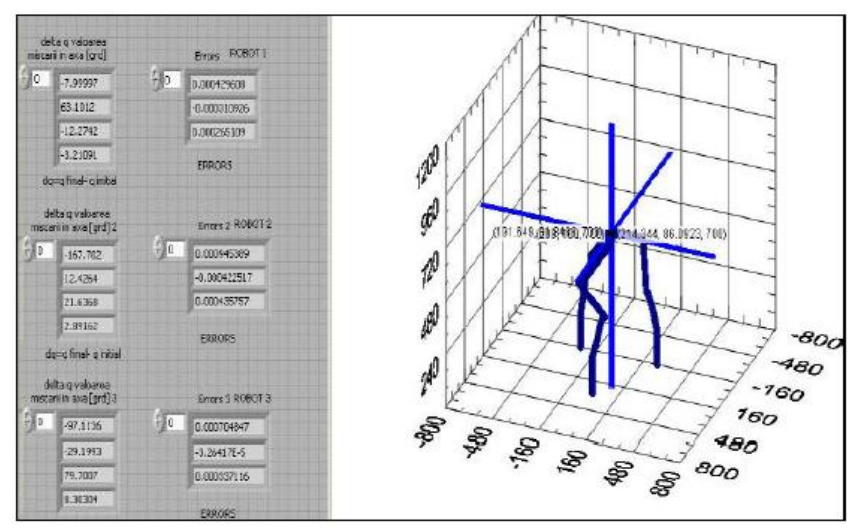

Fig. 8. Simulation of the parallel robot structure with one common point.

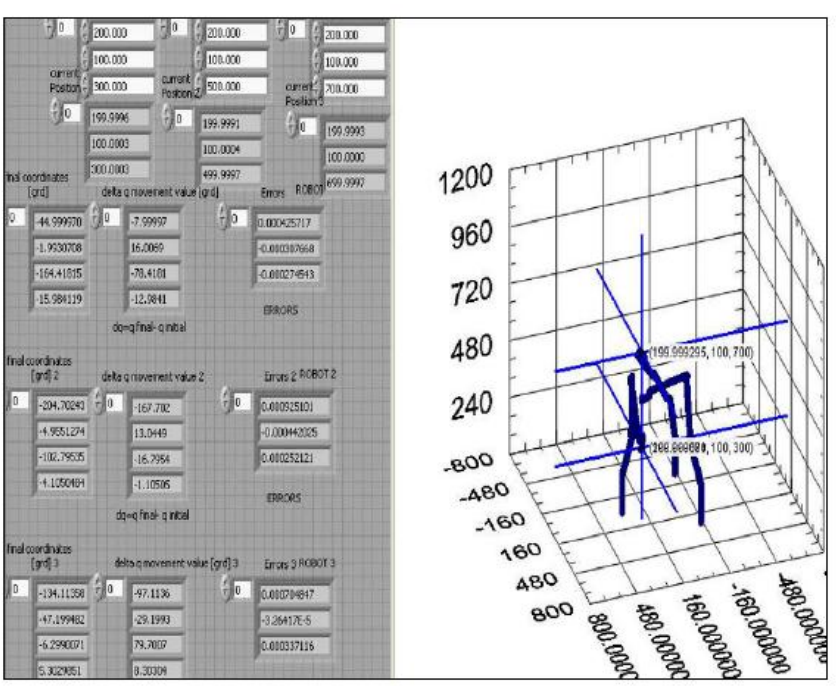

Fig. 9. Simulation of the parallel robot structure with one common line.

As for future work, based on the presented results, we plan to develop more complex LabVIEW VI-s to apply this method to different parallel robot applications in collaborative manner and for different conventional and unconventional space curves.

\section{ACKNOWLEDGMENT}

The results, shown in this paper, were obtained through a mutual research at the Machine and Manufacturing Systems Department, IMST Faculty from University "Politehnica" of Bucharest, Romania, with technical support of Techno Accord Company from Quebec, Canada and Rom Sys SA Mechatronics Company from Romania.

\section{REFERENCES}

[1] S. Kucuk and Z. Bingul, "The inverse kinematics solutions of industrial robot manipulators," IEEE Conferance on Mechatronics, pp. 274-279, Turkey, June 2004, Istanbul, 2004.

[2] S. Kucuk and Z. Bingul, "Robot kinematics: Industrial robotics: forward and inverse kinematics," Industrial Robotics, Theory, Modelling and Control, edited by Sam Cubero,Pro Literatur Verlag, 2006.

[3] J. Denavit and R. S. Hartenberg, "A kinematic notation for lower-pair mechanisms based on matrices", Journal of Applied Mechanics, vol. 1 pp. 215-221, 1955.

[4] J. Funda, R. H. Taylor, and R. P. Paul, "On homogeneous transorms, quaternions, and computational efficiency," IEEE Trans.Robot. Automat., vol. 6, pp. 382-388,1990.
[5] J. J. Craig, Introduction to Robotics Mechanics and Control, USA:Addision- Wesley Publishing Company, 1989.

[6] W. R. Hamilton, "Elements of quaternions," Application of Quaternions to Computation with Rotations, 1979

[7] P. J. Alsina and N. S. Gehlot, "Direct and inverse kinematics of robot manipulator based on modular neural networks," ICARCV, pp. 1743-7, 1994.

[8] J. G. Wang, Y. M. Li, and X. H. Zhao, "Inverse kinematics and control of a 7-DOF redundant manipulator based on the closed-loop algorithm,' International Journal of Advanced Robotic Systems, vol. 7, no. 4, 2010.

[9] R. Manseur and D. Keith, "A fast algorithm for reverse kinematics analysis of robot manipulator," International Journal of Robotics Research, vol. 7, no. 3, 1998, p. 622-648.

[10] C. Welman, "Inverse kinematics and geometric constraints," Master of Science Thesis, Simon Fraser University, Canada, 1989.

[11] D. Gorinevsky and T. Connoly, "Comparations of some neural network and scattered data approximations: The inverse manipulator kinematics example," Neural Computation, vol. 3, no. 6, pp. 521-542, 1994.

[12] L. Lee, "Training feedforward neural networks: An algorithm giving improved generalization," Neural Networks, vol. 10, no. 1, pp. 61-68, 1997.

[13] C. Schittenkopf, G. Deco, and W. Braune, "Two strategies to avoid overfitting in feedforward networks," Neural Networks, vol. 10, no. 3 , pp. 505-516, 1997.

[14] L. C. Wang and C. C. Chen, "A combined optimization method for solving the inverse kinematics problem of mechanical manipulators," IEEE Transaction on Robotics and Automation, vol. 7, no. 4, 1991.

[15] C. Rose, M. F. Cohen, and B. Bodenheimer, "Verbs and adverbs: Multidimensional motion interpolation " IEEE Computer Graphics and Applications, vol.18, no. 5, pp. 32-40.

[16] D. C. C. Wit, B. Siciliano, and G. Bastin, "Theory of robot control," Springer and Verlag, London, U.K, 1996.

[17] L. Zhao and N. Badler, "Gesticulation behaviors for virtual humans," in Proc. the 6th Pacific Conference on Computer Graphics and Applications, pp. 161-168, October 26-28.

[18] A. Olaru, S. Olaru, and N. Mihai, "Proper assisted researchmethod solving of the robotsinverse kinematics problem," Applied Mechanics and Materials, vol. 555, 2014, pp. 135-147.

[19] A. Olaru, S. Olaru, and L.Ciupitu, "Assisted research of the neural network by back propagation algorithm," in Proc. OPTIROB 2010 Conference, Romania, The Reserch Publishing Services Singapore Book, p.194-200, 2010.

[20] A. Olaru, S. Olaru, and N. Mihai, "Application of a new Iterative pseudo-inverse jacobian neural network matrix technique for controlling geckodrive DC motors of manipulators," in Proc. 3rd RSI International Conference on Robotics, p. 114-120.

[21] A. Olaru, "The optimizing space trajectory by using the inverse kinematics, direct dynamics and intelligent damper controlling with proper neural network," in Porc. the 2012 International Conference on Advanced Mechatronic Systems, Tokyo, 2012, pp. 504-509.

[22] A. Olaru, E. Masehian, S. Olaru, and N. Mihai, "Achieving extreme precisions for multiple manipulators using a proper coupled neural network matrix method and LabVIEW instrumentation," in Proc. the 2016 4th International Conference on Robotics and Mechatronics, Tehran, 2016, pp.7-13.

[23] A. D. Olaru and S. A. Olaru, "Assisted optimisation of the robot dynamic behavior with magnetorheological damper," in Proc. the 2009 Fifth International Conference on MEMS NANO, and Smart Systems, Dubai, 2009, pp. 45-49.

[24] C. Gosselin and J. Angeles, "A global performance index for the kinematic optimization of robotic manipulators," ASME J Mech Des 1991, vol. 113, no. 3, pp. 220-226.

[25] T. Yoshikawa, "Manipulability of robotic mechanisms," Int J Robotics Res, 1985, vol. 4, no. 2, pp. 3-9.

[26] J. Salisbury and J. Craig, "Articulated hands: force control and kinematic," Issues. Int J Robotics Res, 1982, vol. 1, no. 1, pp. 4-17.

[27] Y. Li and Q. Xu. "Kinematic analysis of a 3-PRS parallel manipulator," Department Robotics and Computer-Integrated Manufacturing, vol. 23, 2007, pp. 395-408.

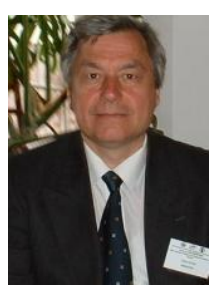

Adrian Olaru finish the University Politehnica of Bucharest, the Faculty of Machine-Tools, Machine and Manufacturing Systems Department. Now, from 1998. $\mathrm{He}$ is a university full professor, and He teaching the following courses are Industrial robots dynamics behavior, LabVIEW application in modeling and simulation of the dynamic behavior of robots and Personal and social robots. He is a doctor from 1989. In the last ten years He has been leading the following 
research projects: computer aided research and design for the hydraulic amplifiers of pneumatic and hydraulic screwdrivers; computer aided research over the dynamic behavior of the forging manipulator orientation modulus; computer aided research over dynamic behavior of the charging manipulators tipping modulus; computer aided research over dynamic behavior of the charging manipulators translation modulus; experimental validation for mathematical models of hydraulic elements and servo system; methodological guide for dimensioning and optimizing electrohydraulic elements; design of the mobile robots; assisted research of the magneto rheological dampers; assisted research of the intelligent dampers; assisted research of the neural networks; optimizing of the robots dynamic behavior by using the Fourier proper analyzer; optimizing the dynamic compliance and global transmisibility by using the assisted research and proper LabVIEW instrumentation; optimize the dynamic behavior and the space trajectory by using the proper neural network.

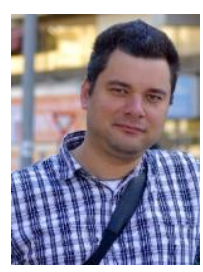

Serban Olaru finished the University Politehnica of Bucharest, Faculty of Machines and Manufacturing Systems, Romania. From 2008 he become the Ph.D. Eng. in the field of mechatronics. Now, he works in RomSYS private company, from Bucharest, Romania, in the department of mechatronics. He write mote than 50 research papers in the fields of intelligent damper systems, mechatronic systems, simulation and modeling with LabVIEW instrumentation.

NiculaeMihai finished the University Politehnica of Bucharest, Faculty of Machines and Manufacturing Systems, Romania. From 2006 he become $\mathrm{Ph}$.D.Eng. in the field of robotics. Now, he is the manager of the private company in mechatronics systems, Technoaccord, Quebec, Canada. 